\title{
New Result on the Critical Exponent for Solution of an Ordinary Fractional Differential Problem
}

\author{
Xinguang Zhang, ${ }^{1,2}$ Lishan Liu, ${ }^{2,3}$ Yonghong $\mathrm{Wu}^{2}$ and Yujun Cui ${ }^{4}$ \\ ${ }^{1}$ School of Mathematical and Informational Sciences, Yantai University, Yantai, Shandong 264005, China \\ ${ }^{2}$ Department of Mathematics and Statistics, Curtin University of Technology, Perth, WA 6845, Australia \\ ${ }^{3}$ School of Mathematical Sciences, Qufu Normal University, Qufu, Shandong 273165, China \\ ${ }^{4}$ Department of Mathematics, Shandong University of Science and Technology, Qingdao, Shandong 266590, China \\ Correspondence should be addressed to Xinguang Zhang; xinguang.zhang@curtin.edu.au
}

Received 20 April 2017; Accepted 22 August 2017; Published 4 October 2017

Academic Editor: Richard I. Avery

Copyright ( 2017 Xinguang Zhang et al. This is an open access article distributed under the Creative Commons Attribution License, which permits unrestricted use, distribution, and reproduction in any medium, provided the original work is properly cited.

We give some background of fractional Cauchy problems and correct an existing result of Cauchy problem for the fractional differential inequality, and we also give an example to illustrate our statement.

\section{Introduction}

It is well known that many engineering and industrial systems involve the convection-diffusion process such as in seepage flows [1], oil flow through porous media in underground reservoirs [2,3], and flow of chemical and metal solutions through porous media in heap leaching $[4,5]$. Traditionally, for the transport of solute in nonheterogeneous porous media, based on the conservation principle and Ficks law, we can use the integer-order differential equation to describe the whole convection-diffusion process. However, it has been established that the convection diffusion equation based on Ficks law fails to model the anomalous feature of diffusion in porous media, and thus fractional order convectiondiffusion equations have the potential to accurately model the convection-diffusion process [6-10]. On the other hand, fractional order integral and derivative operators are nonlocal which can describe the behaviour of many complex processes and systems with long-memory in time. This implies that fractional order models possess much more advantage than integer order models, especially in viscoelasticity, electrochemistry, and porous media [11-15], and many new applications for fractional models in various fields have also been reported recently [16-34].

Fractional Cauchy problems are important in physics to model anomalous diffusion [35], and one has already shown that some transfer processes in a medium and a universal response of electromagnetic, acoustic, and mechanical influence can be described by the fractional Cauchy problem

$$
\begin{aligned}
\left(D_{+}^{\alpha} x\right)(t) & =f(t, x(t)), \quad t>0,0<\alpha<1, \\
\left(D_{+}^{\alpha-1} x\right)(0+) & =b,
\end{aligned}
$$

which has attracted a large number of attention from many researchers and some results on existence of solution have been established in weighted spaces of continuous functions $[36,37]$. However, in the case of lack of regularity, there are very few results for nonexistence of ordinary fractional differential equations and inequalities [26]. Recently, in the absence of regularity, Laskri and Tatar [26] considered the following Cauchy problem for the fractional differential inequality with a polynomial nonlinearity and variable coefficient:

$$
\begin{aligned}
D_{+}^{\alpha} u(t) & \geq t^{\beta}|u(t)|^{m}, \quad t>0,0<\alpha<1, \\
D_{+}^{\alpha-1} u(0+) & =b \in \mathbb{R} .
\end{aligned}
$$

By using the test function method and the integration by parts formula, they established the following nonexistence result with critical exponent separating existence from nonexistence of solutions. 
Theorem 1. Assume that $\beta>-\alpha$ and $1<m \leq(\beta+1) /(1-\alpha)$. Then, problem (2) does not admit global nontrivial solutions when $b \geq 0$.

However, by checking this result carefully one can find that this theorem shows some flaws. In fact, under the above assumptions, problem (2) admits global nontrivial solutions when $b \geq 0$, as shown in the following example.

Example. Consider the following Cauchy problem for fractional differential inequality:

$$
\begin{aligned}
D_{+}^{1 / 2} u(t) & \geq t^{-1 / 6}|u(t)|^{3 / 2}, \quad t>0, \\
D_{+}^{-1 / 2} u(0+) & =I_{+}^{1 / 2} u(0+)=0 .
\end{aligned}
$$

Conclusion. The fractional differential inequality (3) has at least a global nontrivial solution.

Proof. Let $\alpha=1 / 2, \beta=-1 / 6, m=3 / 2$, and $b=0$; then we have $\beta>-\alpha$ and $1<m=3 / 2 \leq(\beta+1) /(1-\alpha)=5 / 3$. And thus the Cauchy problem (3) satisfies all of the conditions of Theorem 1.

But we can easily find that the following function is a global nontrivial solution of the fractional differential inequality (3):

$$
u(t)= \begin{cases}{\left[\Gamma\left(\frac{3}{2}\right)\right]^{2} t^{1 / 2},} & 0<t \leq 1, \\ {\left[\Gamma\left(\frac{3}{2}\right)\right]^{2} t^{-4 / 5},} & t>1 .\end{cases}
$$

In fact, by using the general relationship of RiemannLiouville fractional derivative (see Miller and Ross [27] (p36, 6.6)), we have

$$
D_{+}^{\alpha} t^{p}=\frac{\Gamma(1+p)}{\Gamma(1+p-\alpha)} t^{p-\alpha}, \quad \alpha>0, p>-1 .
$$

Thus we divide the proof into two cases.

Case 1. If $0<t \leq 1$, then

$$
\begin{aligned}
D_{+}^{1 / 2} u(t) & =D_{+}^{1 / 2}\left(\left[\Gamma\left(\frac{3}{2}\right)\right]^{2} t^{1 / 2}\right)=\left[\Gamma\left(\frac{3}{2}\right)\right]^{3} \\
t^{\beta}|u(t)|^{m} & =t^{-1 / 6}\left[\left[\Gamma\left(\frac{3}{2}\right)\right]^{2} t^{1 / 2}\right]^{3 / 2} \\
& =\left[\Gamma\left(\frac{3}{2}\right)\right]^{3} t^{7 / 12}
\end{aligned}
$$

It follows from $0<t \leq 1$ that

$$
\begin{aligned}
D_{+}^{1 / 2} u(t) & \geq t^{-1 / 6}|u(t)|^{3 / 2}, \\
D_{+}^{-1 / 2} u(0+) & =I_{+}^{1 / 2} u(0+) \\
& =\lim _{t \rightarrow 0+} \frac{[\Gamma(3 / 2)]^{2}}{\Gamma(1 / 2)} \int_{0}^{t}(t-s)^{-1 / 2} s^{1 / 2} d s \\
& =\lim _{t \rightarrow 0+} \frac{[\Gamma(3 / 2)]^{3}}{\Gamma(2)} t=0 .
\end{aligned}
$$

Case 2. If $t>1$, we have

$$
\begin{aligned}
D_{+}^{1 / 2} u(t) & =D_{+}^{1 / 2}\left(\left[\Gamma\left(\frac{3}{2}\right)\right]^{2} t^{-4 / 5}\right) \\
& =\left[\Gamma\left(\frac{3}{2}\right)\right]^{3} t^{-13 / 10} \\
t^{\beta}|u(t)|^{m} & =t^{-1 / 6}\left[\left[\Gamma\left(\frac{3}{2}\right)\right]^{2} t^{-4 / 5}\right]^{3 / 2} \\
& =\left[\Gamma\left(\frac{3}{2}\right)\right]^{3} t^{-41 / 30} .
\end{aligned}
$$

It follows from $t>1$ that

$$
D_{+}^{1 / 2} u(t) \geq t^{-1 / 6}|u(t)|^{3 / 2} .
$$

So function (4) is indeed a global nontrivial solution of the Cauchy problem for the fractional differential inequality (3), and the conclusion is proved.

\section{Correction of Theorem 1}

In this section, we firstly point out the flaws in the proof of Theorem 1 in [26] and then give the correct result and the corresponding proof on nonexistence of solution for Cauchy problem (3).

In the proof of Theorem 1 in [26], the authors get the following formulas by defining a test function $\varphi$ and using the properties of Riemann-Liouville fractional integral:

$$
\begin{aligned}
& -\left(I_{+}^{1-\alpha} u\right)\left(0^{+}\right)-\frac{1}{\Gamma(1-\alpha)} \\
& \cdot \int_{0}^{T} \varphi^{\prime}(t) \int_{0}^{t}(t-s)^{-\alpha} u(s) d s d t \\
& \geq \int_{0}^{T} t^{\beta}|u(t)|^{m} \varphi(t) d t, \\
& \frac{1}{\Gamma(1-\alpha)} \int_{0}^{T} \varphi^{\prime}(t) \int_{0}^{t}(t-s)^{-\alpha} u(s) d s d t \leq \frac{1}{m} \\
& \cdot \int_{0}^{T} t^{\beta}|u(t)|^{m} \varphi(t) d t+\frac{\left(\max \left\{1,2^{\beta / m}\right\}\right)^{m^{\prime}}}{m^{\prime}} \\
& \cdot T^{-\beta m^{\prime} / m} \int_{T / 2}^{T}\left(I_{T_{-}}^{1-\alpha} \frac{\left|\varphi^{\prime}\right|}{\varphi^{1 / m}}\right)(t) d t,
\end{aligned}
$$

which corresponds to (11) and (14) in paper [26], respectively. From (10), they obtained

$$
\begin{aligned}
& -\left(I_{+}^{1-\alpha} u\right)\left(0^{+}\right) \\
& +\frac{\left(\max \left\{1,2^{\beta / m}\right\}\right)^{m^{\prime}}}{m^{\prime}} T^{-\beta m^{\prime} / m} \int_{T / 2}^{T}\left(I_{T_{-}}^{1-\alpha} \frac{\left|\varphi^{\prime}\right|}{\varphi^{1 / m}}\right)(t) d t \\
& \geq\left(1-\frac{1}{m}\right) \int_{0}^{T} t^{\beta}|u(t)|^{m} \varphi(t) d t .
\end{aligned}
$$


However, it follows from (10) that the correct form should be

$$
\begin{aligned}
& -\left(I_{+}^{1-\alpha} u\right)\left(0^{+}\right) \\
& -\frac{2}{\Gamma(1-\alpha)} \int_{0}^{T} \varphi^{\prime}(t) \int_{0}^{t}(t-s)^{-\alpha} u(s) d s d t \\
& +\frac{\left(\max \left\{1,2^{\beta / m}\right\}\right)^{m^{\prime}}}{m^{\prime}} T^{-\beta m^{\prime} / m} \int_{T / 2}^{T}\left(I_{T_{-}}^{1-\alpha} \frac{\left|\varphi^{\prime}\right|}{\varphi^{1 / m}}\right)(t) d t \\
& \geq\left(1-\frac{1}{m}\right) \int_{0}^{T} t^{\beta}|u(t)|^{m} \varphi(t) d t,
\end{aligned}
$$

instead of (11), which leads to a wrong result.

In fact, under the assumption of Theorem 1, problem (2) has infinitely many global nontrivial positive solutions; for example, according to the proof of the above example and noticing that $m>1$, for any $\mu \in[1, \infty)$, the following functions

$$
u(t)= \begin{cases}{[\Gamma(\alpha)]^{1 /(m-1)} t^{\alpha},} & 0<t \leq 1, \\ {[\Gamma(\alpha)]^{1 /(m-1)} t^{-\mu-(\alpha+\beta) /(m-1)},} & t>1\end{cases}
$$

are global nontrivial positive solutions of problem (2).

Thus we give the correct statement as follows.

Theorem 2. Assume that $\beta>-\alpha$ and $1<m \leq(\beta+1) /(1-\alpha)$. Then, when $b \geq 0$, Cauchy problem (2) has infinitely many global nontrivial positive solutions. However it does not admit global nontrivial negative solutions.

Proof. Take a test function $\varphi(t) \in C^{1}([0, \infty),[0,+\infty))$, where $\varphi$ is nonincreasing such that

$$
\varphi(t)= \begin{cases}1, & t \in\left[0, \frac{T}{2}\right], \\ 0, & t \in[T, \infty) .\end{cases}
$$

Suppose, on the contrary, that a global nontrivial negative solutions $u(t) \leq 0$ exist for all time $t>0$. Following the proof of [26], we only correct formula (11). By (10), one has

$$
\begin{aligned}
& -\left(I_{+}^{1-\alpha} u\right)\left(0^{+}\right) \\
& -\frac{2}{\Gamma(1-\alpha)} \int_{0}^{T} \varphi^{\prime}(t) \int_{0}^{t}(t-s)^{-\alpha} u(s) d s d t \\
& +\frac{\left(\max \left\{1,2^{\beta / m}\right\}\right)^{m^{\prime}}}{m^{\prime}} T^{-\beta m^{\prime} / m} \int_{T / 2}^{T}\left(I_{T_{-}}^{1-\alpha} \frac{\left|\varphi^{\prime}\right|}{\varphi^{1 / m}}\right)(t) d t \\
& \geq\left(1-\frac{1}{m}\right) \int_{0}^{T} t^{\beta}|u(t)|^{m} \varphi(t) d t .
\end{aligned}
$$

Noticing that the test function $\varphi(t) \in C^{1}([0, \infty))$ satisfies $\varphi(t) \geq 0$ and $\varphi$ is nonincreasing for some $T>0$ and the solution $u(t) \leq 0$ we have

$$
\frac{1}{\Gamma(1-\alpha)} \int_{0}^{T} \varphi^{\prime}(t) \int_{0}^{t}(t-s)^{-\alpha} u(s) d s d t \geq 0 .
$$

Consequently, (15) and (16) guarantee that (11) holds. The rest of proof is similar to those of [26].

Remark 3. Although we correct the result of [26], the accurate critical exponent for solution is still unknown.

\section{Conflicts of Interest}

The authors declare that they have no conflicts of interest regarding the publication of this paper.

\section{Acknowledgments}

The authors were supported financially by the National Natural Science Foundation of China (11571296 and 11371221) and the Natural Science Foundation of Shandong Province of China (ZR2014AM009).

\section{References}

[1] M. Carrizales, L. Lake, and R. Johns, "Multiphase fluid flow simulation of heavy oil recovery by electromagnetic heating," in Proceedings of the SPE Improved Oil Recovery Symposium, Tulsa, OK, USA, April 2010.

[2] E. Cariaga, F. Concha, and M. Sepúlveda, "Flow through porous media with applications to heap leaching of copper ores," Chemical Engineering Journal, vol. 111, no. 2-3, pp. 151-165, 2005.

[3] X. Zhang, L. Liu, and Y. Wu, "The uniqueness of positive solution for a fractional order model of turbulent flow in a porous medium," Applied Mathematics Letters, vol. 37, pp. 2633, 2014.

[4] S. C. Bouffard and D. G. Dixon, "Investigative study into the hydrodynamics of heap leaching processes," Metallurgical and Materials Transactions B, vol. 32, no. 5, pp. 763-776, 2001.

[5] X. Yu and Z. Zhai, "Well-posedness for fractional Navier-Stokes equations in the largest critical spaces," Mathematical Methods in the Applied Sciences, vol. 35, no. 6, pp. 676-683, 2012.

[6] H. Sun, W. Chen, and Y. Chen, "Variable-order fractional differential operators in anomalous diffusion modeling," Physica A: Statistical Mechanics and Its Applications, vol. 388, no. 21, pp. 4586-4592, 2009.

[7] M. M. Meerschaert and C. Tadjeran, "Finite difference approximations for fractional advection-dispersion flow equations," Journal of Computational and Applied Mathematics, vol. 172, no. 1, pp. 65-77, 2004.

[8] S. Fomin, V. Chugunov, and T. Hashida, "Application of fractional differential equations for modeling the anomalous diffusion of contaminant from fracture into porous rock matrix with bordering alteration zone," Transport in Porous Media, vol. 81, no. 2, pp. 187-205, 2010.

[9] X. Zhang, L. Liu, and Y. Wu, "Variational structure and multiple solutions for a fractional advection-dispersion equation," Computers \& Mathematics with Applications, vol. 68, no. 12, part A, pp. 1794-1805, 2014.

[10] X. Zhang, L. Liu, Y. Wu, and B. Wiwatanapataphee, "Nontrivial solutions for a fractional advection dispersion equation in anomalous diffusion," Applied Mathematics Letters, vol. 66, pp. $1-8,2017$.

[11] R. Metzler, W. Schick, H. Kilian, and T. F. Nonnenmacher, "Relaxation in filled polymers: a fractional calculus approach," The Journal of Chemical Physics, vol. 103, no. 16, pp. 7180-7186, 1995. 
[12] J.-P. Bouchaud and A. Georges, "Anomalous diffusion in disordered media: statistical mechanisms, models and physical applications," Physics Reports, vol. 195, no. 4-5, pp. 127-293, 1990.

[13] T. F. Nonnenmacher and R. Metzler, "On the Riemann-Liouville fractional calculus and some recent applications," Fractals, vol. 3, no. 3, pp. 557-566, 1995.

[14] I. Podlubny, "Geometric and physical interpretation of fractional integration and fractional differentiation," Fractional Calculus and Applied Analysis, vol. 5, no. 4, pp. 367-386, 2002.

[15] R. Hilfer, Applications of Fractional Calculus in Physics, World Scientific Publishing Co., Pte. Ltd., Singapore, 2000.

[16] Y. Cui, "Uniqueness of solution for boundary value problems for fractional differential equations," Applied Mathematics Letters, vol. 51, pp. 48-54, 2016.

[17] Y. Zou and Y. Cui, "Existence results for a functional boundary value problem of fractional differential equations," Advances in Difference Equations, p. 233, 2013.

[18] Y. Cui and Y. Zou, "Existence results and the monotone iterative technique for nonlinear fractional differential systems with coupled four-point boundary value problems," Abstract and Applied Analysis, vol. 2014, Article ID 242591, 6 pages, 2014.

[19] X. Zhang and Y. Han, "Existence and uniqueness of positive solutions for higher order nonlocal fractional differential equations," Applied Mathematics Letters, vol. 25, no. 3, pp. 555-560, 2012.

[20] J. R. Graef, L. Kong, and B. Yang, "Positive solutions for a fractional boundary value problem," Applied Mathematics Letters, vol. 56, pp. 49-55, 2016.

[21] X. Zhang, L. Liu, and Y. Wu, "Multiple positive solutions of a singular fractional differential equation with negatively perturbed term," Mathematical and Computer Modelling, vol. 55, no. 3-4, pp. 1263-1274, 2012.

[22] X. Zhang, L. Liu, and Y. Wu, "The eigenvalue problem for a singular higher order fractional differential equation involving fractional derivatives," Applied Mathematics and Computation, vol. 218, no. 17, pp. 8526-8536, 2012.

[23] C. S. Goodrich, "Positive solutions to boundary value problems with nonlinear boundary conditions," Nonlinear Analysis, vol. 75, no. 1, pp. 417-432, 2012.

[24] X. Zhang, Y. Wu, and L. Caccetta, "Nonlocal fractional order differential equations with changing-sign singular perturbation," Applied Mathematical Modelling, vol. 39, no. 21, pp. 65436552, 2015.

[25] X. Zhang, L. Liu, Y. Wu, and B. Wiwatanapataphee, "The spectral analysis for a singular fractional differential equation with a signed measure," Applied Mathematics and Computation, vol. 257, pp. 252-263, 2015.

[26] Y. Laskri and N. Tatar, "The critical exponent for an ordinary fractional differential problem," Computers \& Mathematics with Applications, vol. 59, no. 3, pp. 1266-1270, 2010.

[27] K. S. Miller and B. Ross, An Introduction to the Fractional Calculus and Fractional Differential Equations, A Wiley-Interscience Publication, John Wiley \& Sons, New York, NY, USA, 1993.

[28] X. Zhang, L. Liu, B. Wiwatanapataphee, and Y. Wu, “The eigenvalue for a class of singular $p$-Laplacian fractional differential equations involving the Riemann-Stieltjes integral boundary condition," Applied Mathematics and Computation, vol. 235, pp. 412-422, 2014.

[29] X. Zhang, C. Mao, L. Liu, and Y. Wu, "Exact iterative solution for an abstract fractional dynamic system model for bioprocess," Qualitative Theory of Dynamical Systems, vol. 16, no. 1, pp. 205222, 2017.
[30] F. Sun, L. Liu, X. Zhang, and Y. Wu, "Spectral analysis for a singular differential system with integral boundary conditions," Mediterranean Journal of Mathematics, vol. 13, no. 6, pp. 47634782, 2016.

[31] L. Liu, F. Sun, X. Zhang, and Y. Wu, "Bifurcation analysis for a singular differential system with two parameters via to topological degree theory," Nonlinear Analysis. Modelling and Control, vol. 22, no. 1, pp. 31-50, 2017.

[32] L. Liu, H. Li, C. Liu, and Y. Wu, "Existence and uniqueness of positive solutions for singular fractional differential systems with coupled integral boundary conditions," Journal of Nonlinear Science and Its Applications, vol. 10, no. 1, pp. 243-262, 2017.

[33] L. Guo, L. Liu, and Y. Wu, "Existence of positive solutions for singular fractional differential equations with infinite-point boundary conditions," Nonlinear Analysis, Modelling and Control, vol. 21, no. 5, pp. 635-650, 2016.

[34] L. Liu, X. Zhang, J. Jiang, and Y. Wu, “The unique solution of a class of sum mixed monotone operator equations and its application to fractional boundary value problems," Journal of Nonlinear Science and Its Applications, vol. 9, no. 5, pp. 29432958, 2016.

[35] K. Li, J. Peng, and J. Jia, "Cauchy problems for fractional differential equations with Riemann-Liouville fractional derivatives," Journal of Functional Analysis, vol. 263, no. 2, pp. 476-510, 2012.

[36] K. M. Furati and N.-E. Tatar, "An existence result for a nonlocal fractional differential problem," Journal of Fractional Calculus, vol. 26, pp. 43-51, 2004.

[37] K. M. Furati and N.-E. Tatar, "Behavior of solutions for a weighted Cauchy-type fractional differential problem," Journal of Fractional Calculus, vol. 28, pp. 23-42, 2005. 


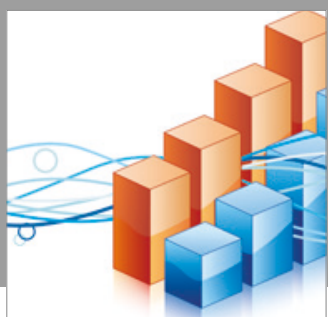

Advances in

Operations Research

vatersals

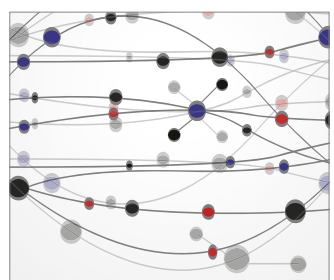

\section{The Scientific} World Journal
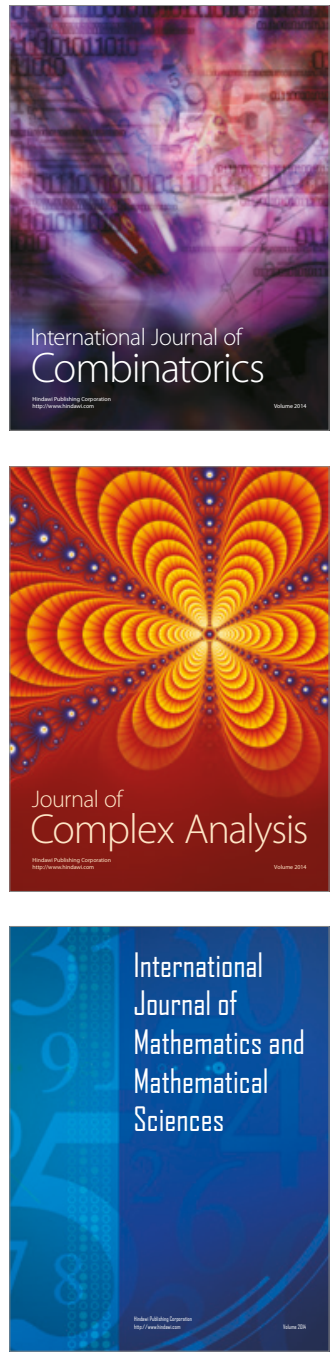
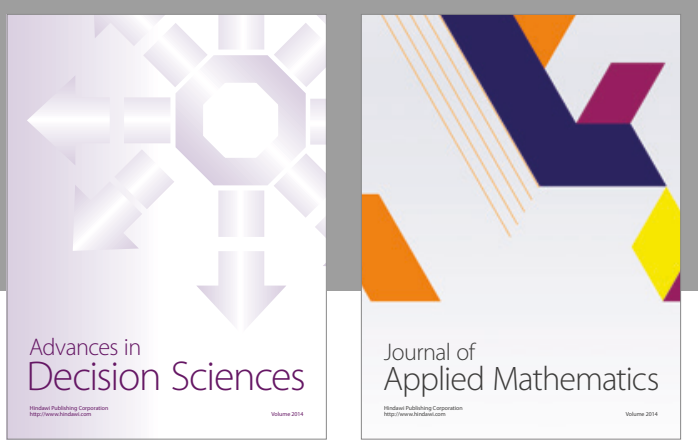

Algebra

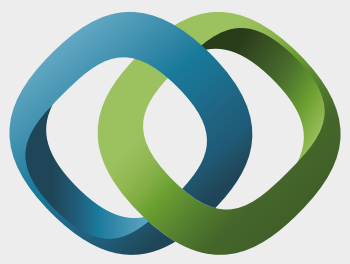

\section{Hindawi}

Submit your manuscripts at

https://www.hindawi.com
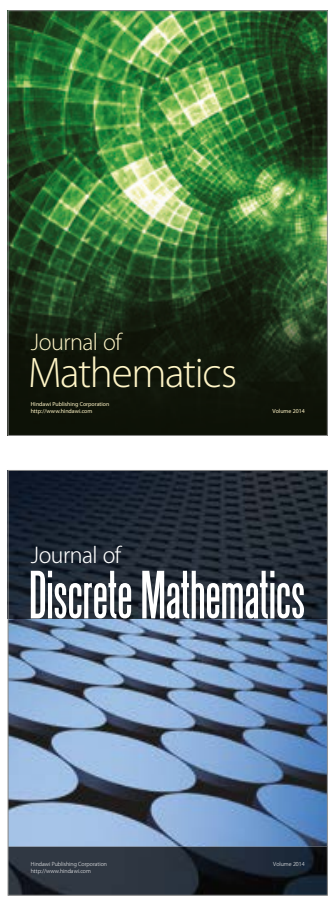

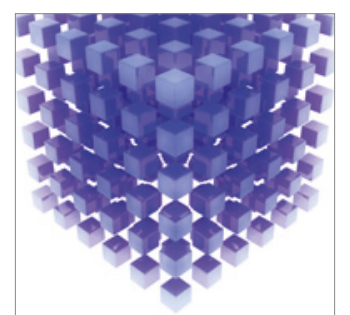

Mathematical Problems in Engineering
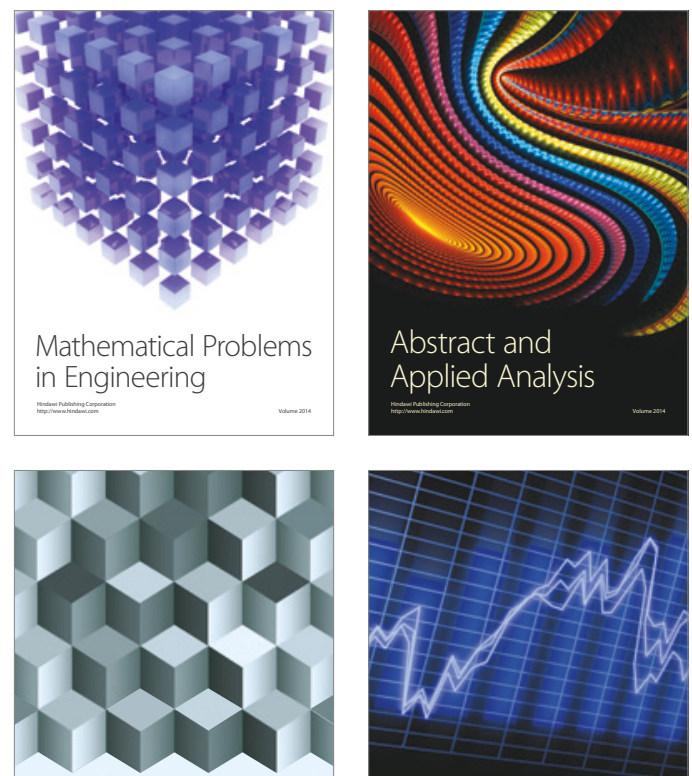

Journal of

Function Spaces

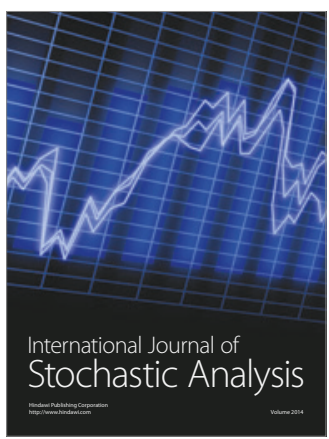

Probability and Statistics
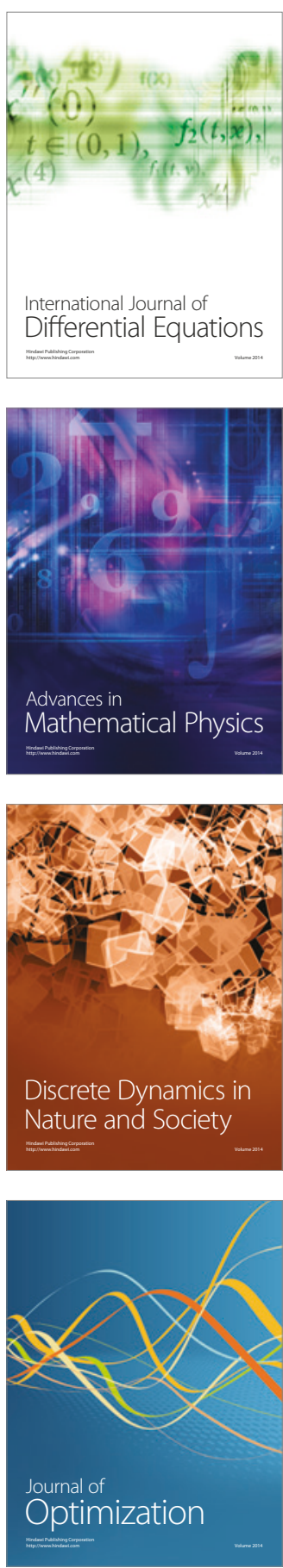